\title{
How Digital Technologies Modify The Work Characteristics: A Preliminary Study
}

\begin{tabular}{|r|l|}
\hline Journal: & The Spanish Journal of Psychology \\
\hline Manuscript ID & SJP--2020-0198.R1 \\
\hline Keywords: & technology, work design, work characteristics, digitalization, digital work \\
\hline Subject Category: & Organizational Psychology \\
\hline Abstract: & $\begin{array}{l}\text { New technologies with unprecedented agentic capabilities (i.e., action } \\
\text { selection, protocol development) are now introduced in organizations } \\
\text { such as Big Data, 3D printing or artificial intelligence. Because they are } \\
\text { endowed with novel capabilities that might compete with human agency, } \\
\text { they might disrupt the way employees work. Based on the work design } \\
\text { model, this study aims to examine their introduction in the daily work } \\
\text { activities and the consequent perceptions of the work characteristics. } \\
\text { Building on Murray's et al., (2020) proposal, we offer a classification of } \\
\text { the digital technologies to conceptualize their relationship with the work } \\
\text { characteristics. To explore the changes induced by two digital } \\
\text { technologies (i.e., drones, robotic automation process), we interviewed } 3 \\
\text { types of employees (i.e., experts, managers, users) from an organization } \\
\text { which has started a digitalization process and we conducted a thematic } \\
\text { analysis. Our analysis revealed three main themes that are discussed: a } \\
\text { technological theme (arresting, assisting), a work characteristic theme } \\
\text { and a theme about the human-technology relationship (agentic, non- } \\
\text { agentic). Results showed that employee autonomy has not been reduced } \\
\text { when digital technologies executed repetitive and unmotivated tasks and } \\
\text { that jobs in the digital work context may be marked by a high level of } \\
\text { knowledge characteristics. Moreover, technologies with agentic } \\
\text { capabilities may be perceived as a non-human agent. Theoretical } \\
\text { contributions for the work design model are then examined. }\end{array}$ \\
\hline & Research Article \\
\hline
\end{tabular}

\section{SCHOLARONE ${ }^{\text {m }}$ Manuscripts}




\section{How Digital Technologies Modify The Work Characteristics: A Preliminary Study}

\section{INTRODUCTION}

Work has an evolving and moving aspect (Howard, 1995; Zuboff, 1988), as evidenced by the diversification of current forms of work (e.g., workers in gig economy) and more flexible work methods (e.g., teleworking) suggesting that the nature of work is changing. Many factors may explain this change in the nature of work: technological advances, growth of international exchanges in the context of globalisation, new organizational models such as "flat" organizations (Grant et al., 2010). Among them, technological advancement has raised a lot of interest as major breakthrough has emerged like the test of a completely autonomous car by Tesla or the development of a robotic hand with high dexterity. These issues shifted from whether technology is automating the activities or informing the worker (Zuboff, 1988) to how far the automation will go (Frey \& Osborne, 2013) and how an efficient collaboration between technology and humans can be conceived (Shneiderman, 2020).

As it has been noted, new technologies can be a double-edge sword for the employee (Ackerman \& Kanfer, 2020), thus it is necessary to think about the consequences of their introduction in the workplace. However, the scientific literature still has little knowledge of how technologies are changing work systems (Barley, 2015). Some research has focused on how technologies are changing employee professional identity (e.g., Eriksson-Zetterquist et al., 2009), relationships at work (e.g., Barley, 2015) or the work context (e.g., McFarland \& Ployhart, 2015). Nevertheless, these studies focused on information and communication technologies (ICT). As pointed out by some authors (e.g., Brynjolfsson \& McAfee, 2014; Schwab, 2017), we have entered a new Industrial Revolution marked by the digital age and characterized by new so-called digital technologies (e.g., artificial intelligence, drones, virtual reality). While the literature knows little about the effect of technologies on work and 
organizations, it knows even less about the effects of digital technologies with some exceptions (e.g., Plesner \& Raviola, 2016; Schwarzmüller et al., 2018).

While it may be difficult to study the effect of technologies in isolation from other drivers of change, the field of work design (WD) as a measure of the micro-context of work (Johns, 2010) allows to examine the effects of technology introduction on work characteristics (WC) (Parker \& Grote, 2020) by capturing the motivational, relational and contextual aspects of work. In addition, some authors point out that changes in organizations make the study of WD even more relevant (Richter et al., 2018; Schneider, 2018). By understanding what is actually changing in employees' work, it will be possible to optimize the introduction of digital technologies (Parker, Morgeson, et al., 2017; Parker \& Grote, 2020).

The objective of this study is to understand the changes that digital technologies can bring in WC using a conceptual framework and to reveal them through interviews. The research questions that motivated the data collection are: 1) what are the changes that the introduction of digital technologies brings in the $\mathrm{WC}, 2$ ) what are the perceived consequences of these changes on their work. Answering Schneider's (2018) call, we used a qualitative methodology to better understand the implications of the digitalization on one's role.

We will first present digital technologies and the conceptual framework we have chosen to study them. Secondly, we will introduce the WD model and suggest some modifications that digital technologies may bring on WC. We will then present the qualitative method followed and illustrate these proposals with the analysis of interviews. Finally, we will conclude by reflecting on the way in which digital technologies influence WD. The contribution of this article is first to display which type of changes digital technologies can bring to WC based on the employee perception of agency in technologies, and second to propose suggestions to the model of WD.

\section{THEORETICAL BACKGROUND}




\section{Digital technologies}

Technologies are drivers of change in organizations (Zammuto et al., 2007). Today, new technologies are introduced in organizations such as Big Data, drones or artificial intelligence and are changing the way of working (Cascio \& Montealegre, 2016). Operators with augmented reality devices which superimpose digital elements on physical objects are appearing in factories. In the maintenance sector, equipment with sensors that send continuous data on their health status allows the emergence of a new form of maintenance (i.e., predictive maintenance) based on the analysis of past data and predictions (Lughofer \& Sayed-Mouchaweh, 2019). In the medical field, hospital staff is confronted with new intelligent assistants (i.e., artificial intelligence) helping them to make diagnoses or propose treatments (Jiang et al., 2017; Powles \& Hodson, 2017).

According to some authors, these advances are part of a new era, the digital era, activated by a 4th Industrial Revolution (e.g., Schwab, 2017). After the steam engine, electricity and ICT (Pozdnyakova et al., 2019), digital technologies could bring radical changes in organizations due to their omnipresence (Cascio \& Montealegre, 2016), complexity and increasing integration (Battistelli \& Odoardi, 2018). The topic of digitalization is of interest to both practitioners (e.g., Schuh et al., 2017; Yoo et al., 2010) and scientists (e.g., Brettel et al., 2014; Waschull et al., 2019) and has received much attention in the last decade (Kipper et al., 2019). The academic community has tried, among other things, to highlight the differences between this 4th Industrial Revolution and the previous one (Parker \& Grote, 2020; Schneider, 2018). In particular, digital technologies seem to be endowed with novel capabilities such as artificial intelligence that can solve problems and learn (Oh et al., 2017). Moreover, while previous technologies can repeat automated sequences according to the way they have been programmed and produce information about the process performed (Zuboff, 1988), digital technologies can introduce variability in the 
repetition of automated sequences by changing their scripts by themselves (Murray et al., 2020). Finally, systems integration could be another property differentiating this digital era (Kohler \& Weisz, 2016 ; Schneider, 2018): whereas previously the different technological systems were not able to connect to each other, digital technologies allow them to exchange information but also ensure communication between people, machines and objects (Hermann et al., 2016).

According to the authors, the technologies that can be labelled as digital differ (e.g., Oztemel \& Gursev, 2018; Petrillo et al., 2018; Salkin et al., 2018). Some attempts to classify them have been proposed (e.g., Inkermann et al., 2019; Wilkesmann \& Wilkesmann, 2018). Among the classifications based on a theoretical framework, the work of Murray et al., (2020) is distinguished by its approach neither totally technology-centred nor totally human-centred (Wang et al., 2020). Their classification is based on the framework of routine (Feldman, 2000) which is seen as both a source of stability and flexibility because the automation of behaviors frees up cognitive resources to find new ways of doing things. The authors examine in detail what constitutes routine (i.e., protocol development, action selection), integrate the notion of agency, and develop the concept of conjoined agency that characterizes the shared capacity for humans and non-humans to exercise intentionality. By distinguishing the locus of control for the two components (either human or technological), four types of conjoined agency are identified: with assisting technologies, augmenting technologies, arresting technologies, and automating technologies. The impact of each type of technology on routine change, on the predictability of routine change and on routine responsiveness is then examined. This useful framework gives through examples indications of the digital technologies that can lead to a type of conjoined agency but does not classify the different technologies. Although this may be difficult because the effects of technologies are dependent on the managerial choices associated with their introduction (Parker, Van den Broeck, et al., 
2017), researchers have noted however the need for a conceptual framework to structure the discourse around digital technologies to better understand their impacts (e.g., Hofmann \& Rüsch, 2017). Therefore, based on this work, we propose a classification of digital technologies (some encountered in the course of our research, others that we have not investigated) (Figure 1). A first step was to bolster the definitions of the different categories developed by the authors in order to classify the digital technologies. The classification of these technologies is based not only on what they allow (i.e., functionalities) but also on what they enable the employee to achieve with them (i.e., affordances) (Zammuto et al., 2007).

(Figure 1 about here)

Assisting technologies. They can be defined as technologies that support the employees in carrying out tasks and leave them freedom of decision. For Murray and colleagues (2020), this type of technology is not agentic: the employee has the expertise and controls the technology while the technology does not determine what to do. Drones are technologies completely handled by pilots. Pilots control with a tablet or a smartphone the flying machine, the flight plan, the images and videos taken. Drones help by allowing the pilot to easily access hard-to-reach places (Irizarry et al., 2012). Immersive virtual reality devices allow people to move independently in a fully simulated reality (Suh \& Prophet, 2018). These devices are widely used for training. During a training activity, the learner can perform the right gestures and learn organizational procedures based on feedback. Finally, exoskeletons are a kind of artificial prosthesis that supports and accompanies the employee's gestures (Lupushor \& Fradera, 2017) so that the employee performs them in an appropriate posture, thus limiting physical risks. Exoskeletons are not robots and do not perform any movement without being initiated by the employee.

Augmenting technologies. They do not reproduce sequences of human action but can develop other sequences which they submit to the human agent. They offer active assistance 
by making recommendations and thus possess a certain degree of agency but do not establish what to do. They are called augmenting technologies probably because they increase one's capacities and senses. Analytical technologies (i.e., Big Data, structured artificial intelligence) that collect, process, analyse, and make inferences about large volumes of data can be classified in this category (Salkin et al., 2018). They thus complement human practices by detecting trends in these data from numerous and complex computations that are humanly difficult to perform, by proposing recommendations based on these analyses, and by leaving the final word to the human agent. Augmented reality devices add virtual elements to physical reality, and can detect and scan objects present in the environment in order to provide relevant information to guide action or offer technical support (Jetter et al., 2018). It does not determine what to do but executes vocal or gestural commands given by the human agent.

Arresting technologies. These technologies select and implement pre-planned actions when preset conditions are met. They are limited by their program to specific procedures and tasks and shall not deviate from them. A human-technology collaboration can take place where humans delegate the task to the technology and the technology stops when the conditions for implementing the program are not met and lets the management of exceptions to humans. The authors of the classification include the blockchain in this category. In particular, blockchains are used for contract elaboration between actors and are governed by rules, established by the actors or creators of the blockchain (Mendling et al., 2018). Some are based on "if-then-else" algorithms, as is the case with robotic process automation (RPA). While blockchains can be used for procedures at the inter-organizational level, the application of RPA is at the task level (Mendling et al., 2018). Contrary to its name, RPA is not a physical robot but a program that uses the same interfaces as the employee and reproduces the sequences that the employee performs. Usually, the program code tries to anticipate the most common situations encountered and the subsequent actions to be taken. Thus, human action is 
only required when the situation encountered has not been anticipated. Finally, the $3 D$ printer is a versatile production system that involves neither tools nor moulds except the printer and which can produce a physical object from a 3D model (Weller et al., 2015). It is able to produce the object from the 3D model and the preset sequence, but cannot define autonomously the quantity to produce or the actions that have to be taken to improve quality.

Automating technologies. Like arresting technologies, this type of technology is able to select actions, but it is programmed to perform a sequence of actions without the need for human intervention. Moreover, like augmenting technologies, these technologies are able to develop new ways of doing things by collecting and analysing a large amount of data. These technologies are equipped with learning capabilities that enable them to correct the analyses they perform and optimize work processes. It is therefore possible that the sequences of action they are able to perform may change as they learn. For the authors, they substitute the human agent insofar as they can manage routine practices. Artificial intelligence based on unstructured data (i.e., which can process other types of data than data frames) can be classified in this category. It differs from artificial intelligence based on structured data by its independence in information retrieval, protocol formulation and execution, and learning. This independence has setbacks since it can lead to the development of poorly performing protocols. Applications of this artificial intelligence can be seen in autonomous production systems or automated guided vehicles (AVGs) operating in workshops or warehouses. In the latter example, the program learns the shop floor plan and calculates the fastest way to get from one point to another. In warehouses such as Amazon's, the program can simultaneously manage hundreds of autonomous vehicles carrying goods to optimize each vehicle's way based on others.

It should be noted that this suggested technology classification is not a fixed framework. The categorization of technology depends on its purpose, the functionalities that 
are integrated and its use. Moreover, digital technologies are characterized by their increasing interconnection, so it is possible that the implementation of a digital technology may be accompanied by the joint introduction of other technologies. Therefore, one might consider their overall effect on work instead of their separate effects.

\section{Work Design Model}

Any impacts that these technological changes may have on work can be captured within the framework of WD (Parker \& Grote, 2020). Indeed, it captures "the content and organization of one's work tasks, activities, relationships and responsibilities" (Parker, 2014) and highlights WC that motivate employees. WD models postulate that WC contribute to job satisfaction and performance (Hackman \& Oldham, 1975) which is supported by metaanalyses (Fried, 1991; Humphrey et al., 2007). Although the original WD model only took into account elements relating to individual tasks, the models developed successively sought to answer the limitations addressed and were enriched by multidisciplinary work (Campion, 1988). Thus, the most complete model validated to date entails four main categories and twenty-one sub-dimensions (Morgeson \& Humphrey, 2006). Task characteristics refer to "how the work itself is accomplished and the range and nature of tasks associated with a particular job" (Morgeson \& Humphrey, 2006, p.1323). They include the dimensions developed in the original model (Hackman \& Oldham, 1975), namely autonomy, task significance, task variety, task identity and feedback from the job. Knowledge characteristics refer to the cognitive processes, skills and abilities involved in the work and include the dimensions of complexity, specialization, problem solving, information processing and variety of skills. Social characteristics relate to the interpersonal dimensions of work such as interdependence, feedback from others, social support and interactions outside the organization. Finally, the work context characteristics relate to the elements that make up the work environment and the physical efforts that one has to make. They include physical 
demands, working conditions, ergonomics and equipment use. A meta-analysis showed that this enriched model explains some of the variance in results beyond traditional characteristics (Humphrey et al., 2007).

The WD literature has shown that technologies influence the way work is designed both directly and indirectly through managerial decisions about their introduction (Kemp \& Clegg, 1987; Parker, Van den Broeck, et al., 2017). Technologies put significant constraints on WD and thus constitute contingency factors (Morgeson \& Campion, 2002). Different technologies have been studied by WD researchers. For instance, Parker and Wall (1998) studied the implementation of modern manufacturing technologies (e.g., just-in-time production, total quality management, advanced manufacturing) and hypothesize that these technologies enhance cognitive demands, accountability and interdependence. They suggest that in uncertain work contexts, decentralisation of decision-making should be favoured in order to control variations at their source. More recently, Wang et al., (2020) have reviewed the effect of ICTs on WC and highlight mixed effects on work demands, autonomy and social relations. Finally, in the context of digitalization, Waschull et al., (2019) made hypotheses regarding the modification of WC. These studies provide insights into how digital technologies could affect WC. Nonetheless, the scientific literature highlights the need to update models to reflect changes in the nature of work (McFarland \& Ployhart, 2015; Oldham \& Hackman, 2010). Some research has focused on the WC of workers in the gig economy (e.g., Deng \& Joshi, 2016; Schroeder et al., 2019) while others have focused on how virtuality affects WC (e.g., Gibson et al., 2011). These have led to the identification of new contextspecific WC, which is why the aim of this study is to understand the modifications of digital technologies on the already-known WC of work, but also to highlight new characteristics specific to the digital work context. Based on the development of the previous typology, we will suggest some modifications induced by the different types of digital technology on the 
WC established by the literature. Table 1 represents a summary of our propositions. Then we will try to highlight potential new features of the work on the basis of the literature.

Task characteristics. Autonomy is a fundamental task characteristic. Indeed, the different conceptual and theoretical frameworks have always given a crucial place to autonomy (i.e., the level at which the employee has the freedom to decide in his work) as it is a fundamental need of individuals ( i.e., self-determination need, Deci \& Ryan, 1985). It might reduce the deleterious effects of organizational change (Trist \& Bamforth, 1951) and is seen as a resource for preserving employee health (e.g., Demerouti et al., 2001; Karasek, 1979). In the model, autonomy consists of three sub-dimensions: work method, work scheduling and decision-making autonomy (Morgeson \& Humphrey, 2006). Empirical work has shown that technologies allow employees to decide when, where and how they work (Xie et al., 2018), thus contributing to the increasing perception of autonomy among employees. However, these technologies may constrain the level of autonomy by allowing the monitoring of employee actions through electronic surveillance devices (e.g., Sewell et al., 2012).

The perceived autonomy can be directly influenced by the sharing of action selection and protocol development between human and technology. Indeed, action selection can relate to autonomy in decision-making and is the means through which intentionality is expressed. Intentionality is a fundamental property of agency; through action plans and strategies to achieve them, people seek to influence their environment (Bandura, 2008). Protocol development can relate to work method autonomy. As with action selection, the development of protocols is a means by which the employees exercise control over and modifies their environment. When these capabilities are offered to technology then humans can feel that they are not the origin of actions since they don't decide. The more human agents answer to the actions prescribed by the technology, the more their sense of self-determination at work may diminish. In many situations the employee is not always agent and this has few 
consequences. However, when employees work with technologies that appear to be invested with human capabilities (i.e., decision support), biases may appear (Parasuraman \& Manzey, 2010; Skitka et al., 2000). In particular, Skitka et al., (2000) highlighted that the employee can make mistakes by following incorrect procedures or recommendations from technology and not checking them. In investing the psychological mechanisms, they noted that this bias can be explained by the belief in the superiority of technological judgment over human judgment. This mechanism could be reinforced by the belief that technologies are objective and therefore capable of making decisions as fair and trustworthy as managers (Lee, 2018). These studies show the drawbacks of non-agency in work situations: if the technology has the ability to select actions and develop protocols, then the human agents can rely on the actions taken or recommended by the technology and reduce their involvement in tasks.

Based on these elements, it seems that technologies that do not have these abilities have little influence on the employee autonomy. When technologies are endowed with agentic traits (i.e., protocol development or action selection), autonomy can diminish because the employees are likely to develop a bias that makes them rely more on the technological judgments and suggestions than on their own judgments. Technologies that are equipped with both capabilities substitute the human in the task, so employees working in the substituted jobs can perceive a total replacement and thus a strong decrease in their autonomy.

Proposition 1: Digital technologies with agency capabilities (i.e., augmenting, arresting, automating) will change the perception of autonomy

Knowledge characteristics. The level of knowledge appears to be increasingly important in work due to the growth of a knowledge-based industry and the increasing complexity of jobs with more cognitive demands (Grant et al., 2010). Indeed, work demands in the post-industrial information age are more cognitive than physical (Howard, 1995). Research has shown that ICTs can induce more cognitive demands (e.g., information 
overload) but also new demands at work (e.g., demand for continuous learning, multitasking) (e.g., Saunders et al., 2017; Xie et al., 2018). The Eurofound (2018) study showed the evolution of four industrial jobs in which new technologies have been implemented. The results showed, among other things, an increase in intellectual tasks in these jobs. For example, employees must now document incidents and problems encountered or assess progress towards achieving objectives.

While technologies can perform low complexity tasks, they can increasingly handle complex tasks (e.g., Battistelli \& Odoardi, 2018; Parker \& Grote, 2020). According to Waschull et al., (2019), they can manage the functions of human information processing (i.e., information acquisition, information analysis, decision selection, decision implementation) as well as innovation. They can therefore substitute humans for tasks involving information processing and problem solving.

Technologies with only the ability to select actions can autonomously perform some defined procedures. Tasks difficult to program (e.g., with many exceptions) are always performed by humans. This can result in a distribution of work where technology performs repetitive and easily programmable tasks and humans are left with the more complex tasks that are difficult to program. If humans must manage exceptions, then they must mobilize their knowledge and expertise and process information about the problem to complete the situation. At the same time, these repetitive tasks can also be unattractive for the employee as they involve the execution of a routine procedure and rarely lead to skill development.

Technologies that can only develop protocols will not increase the complexity of the tasks since they take care of analysing the data and provide the employee with understandable information on which to base decisions. They also help to solve problems by finding previously unknown relationship patterns and thus finding possible solutions. They can therefore carry out some of the characteristics of complexity, information processing and 
problem solving, which might be detrimental for the employee. Indeed, these characteristics can contribute to the development of self-efficacy, which is itself essential to the development of agency: by placing employees in increasingly complex situations adapted to their skills, they gradually acquire the confidence of being able to perform successfully in a given situation (Bandura, 1997). This sharing may reduce the situations in which the employees are confronted with mastery experiences that stimulate their self-efficacy. In addition, by discovering new relationships, these technologies can propose improvements to existing protocols and thus be a source of innovation and change. Nonetheless, by bringing some changes, individuals can influence their environment and exercise their power to act. Also, if these functions are taken over by technology, the employees may use their power to act less often and in the long run may feel unskilled because the employees no longer use and reinforce them as often and as extensively.

The impact of these technologies on knowledge characteristics may also depend on the managerial choices made. If managers legitimize technologies carry out these characteristics by favouring the solutions or the suggestions they provide to the detriment of human suggestions, this will encourage less in the long-term one to find solutions at work. Indeed, employees may give up making efforts if they believe that no matter how hard they will try, they will not produce results (Bandura, 1982; Seligman, 1972).

When technologies are equipped with both capabilities, they can reproduce all human information processing functions and thus totally substitute the human, regardless of task complexity and program complexity. Thus, they can perform simple, complex and routine tasks by learning, adjusting and optimizing their analyses. Employees will then be assigned supervisory tasks or tasks on which technologies have not yet been trained. The same dilemma then arises as with modern manufacturing as to whether this monitoring will involve sustained attention and vigilance to anticipate problems or whether it will involve a passive 
attitude in that the technology itself can anticipate the occurrence of problems (Parker \& Wall, 1998). It is likely that with their new analytical capabilities, the technologies are able to predict and anticipate their occurrence. For employees working in the jobs they have substituted, this change may have a strong influence on the knowledge characteristics of their work.

Proposition 2: Digital technologies with agency capabilities (i.e., augmenting, arresting, automating) will change the perception of knowledge characteristics

Social characteristics. Social relationships correspond to another fundamental need for individuals (i.e., relatedness, Deci \& Ryan, 1985). The interrelations have been multiplied, facilitated and modified with ICTs and social media which allow for synchronous or asynchronous answers from any location while keeping track of these exchanges (McFarland \& Ployhart, 2015). Digital technologies may continue to change these interactions as one of the fundamental characteristics of digitalization is the interconnection of systems (Kohler \& Weisz, 2016). Whereas previous systems and equipment did not "communicate" with each other and did not synchronize, they now have a high degree of connection by constantly exchanging information (Schneider, 2018). Interconnection between technologies could affect human systems, in particular by increasing exchanges and interdependence between members of different departments of the organization or between organizations. Indeed, exchanges would be less constrained by accounting issues, as systems integration would create a bridge between two technologies that do not necessarily belong to the same organizations.

The model recognizes two forms of interdependence: initiated (i.e., the extent to which work moves from one job to other jobs) and received (i.e., the extent to which a job is affected by the work of other jobs) interdependence (Morgeson \& Humphrey, 2006). While interdependence and interactions outside the organization relate to the opportunities for the employee to interact and therefore the quantity of social interactions at work, social support 
relates to the quality of these interactions (Wall et al., 1990). Feedback from others can be studied both in terms of quantity (i.e., frequency) and content (Ashford et al., 2016).

Digital technologies could change the chains of interdependence through the action selection of actions and the development of protocols. If technology is capable of performing a whole sequence of actions by itself, humans will be able to use it for defined tasks. When the task can be handled by the technology, it is likely that employees will delegate it to the technology and rely less on a human colleague to perform it. The chain of interdependence may thus change for programmable tasks: the initiated interdependence may decrease because the work will pass less from human to human. For tasks difficult to program, the chain of interdependence is more likely to remain the same as complex tasks require more knowledge, skills and resources (Wood, 1986) and interdependence helps to share and to combine them. If the technology can develop protocols, it can provide humans with new ideas and recommendations. The received interdependence will be further impacted because human work is likely to be impacted by the input of technology. In addition, the employee may develop a bias when interacting with technology as the employee has more confidence in the suggestions offered by the technology. This bias may lead the employee to look less for solutions or new ways of doing things and to interact less with others to find them.

With both capacities, the influence on interdependence could be cumulative: the work input could come from technology and a wider range of tasks could be delegated to it. By replacing the human, there would potentially be fewer employees working around the technology and therefore fewer potential contacts. Fewer potential contacts also reduce the possibility of being offered social support (Wall et al., 1990).

Proposition 3: Digital technologies with agency capabilities (i.e., augmenting, arresting, automating) will change the perception of social characteristics 
Work context characteristics. Work context characteristics have been less investigated by researchers (Morgeson \& Humphrey, 2006) but are presumed to be more related to stress (Humphrey et al., 2007). Regarding the evolution of work, their relevance to more cognitive and virtual jobs, to less manual jobs, and to more sedentary jobs is of interest. Moreover, the ergonomics of jobs can be improved with lifting robots or exoskeletons (Spada et al., 2017) and with the directives related to hygiene, quality and safety that are becoming standards in companies. It is then possible that these contextual conditions of work may become minimum work standards in the future.

Schroeder et al., (2019) proposed that new contextual characteristics be developed to account for the working conditions of digital platform workers. In particular, they suggest taking into account the quality of the equipment and technologies used. This characteristic may also apply to work in organizations as this quality may differ from one service to another in terms of the quality of the internet connection (e.g., employees working on the field) or the quantity of equipment available (e.g., an insufficient number of tablets for the team). All of these aspects can impact employee performance. For the moment, the model only takes into account equipment use which can also be modified because of the intensity of technological use at work.

Proposition 4: Digital technologies with agency capabilities (i.e., augmenting, arresting, automating) will change the perception of equipment use

New work characteristics. Some authors have focused on the impact of technologies on WD (Deng \& Joshi, 2016; Xie et al., 2018). Xie et al., (2018) proposed new characteristics called hybrid characteristics (i.e., multitasking, demand for constant learning, non-workrelated interruptions, boundarylessness) because they are assumed to be non-classified in a single main category. Deng and Joshi (2016) tested whether WD characteristics are still relevant for workers on digital platforms and highlighted motivational characteristics specific 
to this context (e.g., digital work control, payment for micro-tasks). We draw inspiration from the latter approach to ensure that already-known WC are still as important to employees in the face of the digitalization of activities and to identify emerging characteristics of this new work context.

In particular, we believe that digital technologies will greatly bring some modifications on social characteristics. Indeed, while ICTs can increase connections between employees by enabling instantaneous and asynchronous communication with people in different locations (McFarland \& Ployhart, 2015), digital technologies can enable new forms of interaction by also offering immersive experiences with tactile, auditory, visual stimuli, etc. The increasing integration of systems could also affect the relationship between technologies and humans. Indeed, this leads employees to interact more with technologies like collaborative or "social" robots (Broadbent, 2017), telepresence robots (Koceski \& Koceska, 2016) or artificial intelligence (Brynjolfsson \& McAfee, 2014). Moreover, interactions between humans and technologies are becoming more complex: technologies can answer voice commands, hold a conversation, and can learn as they interact with humans (Brenon et al., 2016; Rubin et al., 2010). The scientific literature has shown that daily interactions with robots contribute to the development of emotional attachment and perception of mind; this is more likely when the technologies are humanlike and can exhibit social cues (e.g., gaze, proxemic behaviors) (Broadbent, 2017; Fiore et al., 2013). Based on these empirical facts, it can be assumed that employees may attribute human traits to technologies that can select actions and develop protocols because these technologies express features of human agency, respectively intentionality and the possibility of influencing the work environment, and that this will be more likely for technologies that have a humanoid form or that can send social cues (e.g., having a conversation). However, the literature knows little about how these 
attributions may impact the relatedness need of human beings and thus their perceptions of quantity and quality of relationships at work.

Proposition 5: Digital technologies with agency capabilities (i.e., augmenting, arresting, automating) will bring modifications in social interactions at work

(Table 1 about here)

\section{METHOD}

The aim of our study is to explore and get a preliminary understanding of what is really changing in work activities. Therefore, qualitative method appears to be the best option as it allows researchers to have a rich, unstandardized and detailed data set (Howitt, 2016). Qualitative methodology provides several objectives from description to theoretical development with specific analysis better suited for each of them. Because our study aims at describing a phenomenon and at developing new concepts, more complex analyses (e.g., grounded theory) suited for theorizing were not performed. We used interviews to collect data as it permits for the interviewer to ask for supplementary explanations and clarifications. We investigated drones and RPA to explore technologies considered as not agentic (drones) and agentic (RPA) and to highlight some differences. Moreover, robots have been introduced in industrial jobs for decades but today robots (i.e., RPA) are introduced in the business which is relatively new.

\section{Context and sample}

The organization is a large national Belgian company in the transport sector. The company has initiated a digital transformation process in 2018 by integrating digital in its strategy. Since, the company has gradually digitalized its activities. Transports appear to be a great research field as digitalization may bring many opportunities and challenge for this sector (Davidsson et al., 2016). As, previous research has shown transport sector is an industry characterized by safety climate (Díaz \& Cabrera, 1997; Flin et al., 2000), therefore 
security represents an important outcome and some technological changes have been implemented in the past few years to maximize it. The company's interests in this study are to understand the human issues and to have some directions in the digital transformation.

We identified with the team project some digital technologies which were developed. Among these technologies, two had reached the implementation phase and had some impacts on the employee activities: drones and robotic automation process (RPA). Contrary to drones, RPA's implementation was limited to a single service (i.e., accountability), thus the introduction of these two technologies was quite different. Volunteers of the accounting department were put as a team and received from an external consultant a five-days training in programming (three days with an e-learning and two days with an instructor). The training aimed at offering employees coding basics to develop themselves some of the RPA's programs. The newly developers from the accounting department worked on their own program and met weekly to review what each was doing and helped each other. They were accompanied by some IT employees to support and supervise their progress. At the time of this study, some of the programs were launched whereas others were not. This technology can be used by the entire department and thus will change many aspects for the activities.

Drones were displayed in 2016 but this technology is not deployed evenly. In some regions, drones are more frequently used because of their geographic profile. Another reason is because the implementation depends on the employees. The utilization of drones is voluntary and employees have to convince their superiors of their benefits for the service, and then have to pass an exam to become a pilot. The initial function of pilots can vary a lot, and the impact on jobs may differ based on what employees are used to do.

\section{Procedure}

We conducted 9 semi-structured interviews which composes our data corpus. We interviewed 3 types of employees: IT employees who have some knowledge about it (here in 
after called experts), managers who might have noticed some changes and employees whose work changes with the technology (here in after called users) (Table 2). First, we interviewed experts and managers, and then users were identified by them. Each interview lasts approximately between 45 and 80 minutes. Interviews were carried out either face-to-face or remotely via videoconference or telephonic call. The participants were asked about their work and their activities to have a glimpse of their work tasks, then about the technology and the changes it brought. Finally, they were asked about the appraisal of these changes (see Appendix). When a participant did not talk about an aspect identified through literature as possibly being impacted (e.g., autonomy, specialisation, information processing), we asked them about it.

\section{(Table 2 about here)}

Our data set has been identified "by a particular analytic interest in some topic in the data, and [the] data set then becomes all instances in the corpus where that topic is referred to" (Braun \& Clarke, 2006). Thus, we identified in our data corpus all occurrences which deal with the changes in work owing to digital technologies. Each interview was recorded and transcribed. Then a thematic analysis was proceeded through the software QDA Miner ${ }^{1}$ as it represents a good approach to describe a phenomenon (Howitt, 2016). It corresponds to "a method for identifying, analysing, and reporting patterns (themes) within data" (Braun \& Clarke, 2006). It is depicted as a flexible research tool because it is affiliated to particular theoretical orientation (Howitt, 2016). However, it requires the same rigour as other qualitative methods in that steps are quite similar. Scholars can be quite sceptical about this analysis because it presents many drawbacks, especially the fact that the researcher often gives little detail of how he or she carried out the analysis and thus limiting the understanding

\footnotetext{
${ }^{1}$ Interested readers may refer to O'Kane et al., (2019) to get an insight of this software and others of its kind and how they can be used
} 
of choices made and the discussion. Braun and Clarke (2006) detailed the steps to conduct a good thematic analysis and thus improve the quality of studies using it. Following their guidelines, we conducted a theoretical thematic analysis based on Morgeson and Humphrey's (2006) framework. Based on realistic assumption, we chose to focus on the semantic content of the data. We decided to proceed with a theory-driven coding. Therefore, we approached our data set with our research question in mind. As a reminder, the aim of the study is to identify the common topics about WC when employees are confronted by the introduction of digital technologies in their work. For coding, we used the code and comment functions of the software. After the initial coding where codes can apply to different themes, we combined them to sort potential themes by examining how codes can be assembled and how they are part of a bigger point. No quantitative method was used to sort the themes. Then we reviewed, refined and labelled them. As proposed by the authors a thematic map might be helpful to clear up the analytic process (Figure 2). In our analysis, we identified three themes: a descriptive theme around technologies, a theme about the consequences on work and a theme dealing with the human-technology relationship. The first theme helps to build a case to classify the two technologies into different categories. Finally, verbatim were extracted by us regarding their relevance and how well they illustrate the report.

\section{RESULTS}

\section{Classification of the technologies}

\section{$R P A$ as an arresting technology}

RPA is not a physical robot but a program. It works like a macro that is a program which executes commands automatically and reduces the operations performed by the user. In the company, the robot is limited in execution to one task at a time, is able to manage priorities and gives a report of its actions. It is easy to use and has documentation to know how to use it. It also has the ability to communicate with different systems: whereas 
previously humans had to manually encode information from one system to another, the robot is now able to do this automatically.

The technology can replace and perform what the human does for routine and standardized tasks - those well-defined step by step (e.g. logging into a system, opening an email). It is unable to deviate from the programmed procedures and steps. When the situation varies from its program, the technology stops and sends a message to the worker to indicate that the task has not been completed. The technology therefore cannot handle the exceptions that are left to humans. The program of the technology is modifiable. As processes and procedures evolve, programs must evolve. The program can also be modified to improve it when the results produced are not satisfactory. Writing the program and automating the task is therefore an iterative process.

"The robot cannot make choices or find its own way. You have to tell it everything, it doesn't know anything in advance so you really have to detail at the very beginning the process to execute and it has to be quite standardized because when there's something it doesn't know or doesn't recognize, it stops". (Participant 1, manager) ${ }^{2}$

RPA can perform the following tasks: cost search, invoice creation or transfers. Cost search involves extracting data, combining data from different systems and synthesizing the extracted information. These operations are performed faster by technology than by humans. Technology can also detect problems by highlighting discrepancies between data from different systems. For cost search, technology does the preparatory work by providing relevant information that allows the worker to identify problems more quickly and to implement actions. The worker therefore carries out the interpretation and verification of the information. In the context of transfers and invoices, the technology automatically encodes the information into the system instead of the worker.

\footnotetext{
2 The interviews were all in French. We chose not to translate them into proper English but to translate them literally to keep the meaning of the participant's discourse.
} 
RPA developers experience different changes in their work that those experienced by users. It is a whole new role for developers, that brings a task conflict between the requirements of the two roles (i.e., accounting, development team). For developers, it was difficult to combine the schedules and meet both expectations.

\section{Drones as an assisting technology}

Drone is a kind of remote-controlled and remotely guided helicopter. It can be equipped with different sensors (thermal cameras, shooting camera) that change its functionalities. It allows to access difficult places and to take videos and images. In the company, it is used for different purposes: realization of work plans, photogrammetry, inspection or communication reports. At the time of this study, drone is not autonomous in the company, although autonomous drones are developing on the market. Drone comes with several constraints. First, there are meteorological constraints because drones cannot be used when it rains or winds.

The introduction of drones in the company is not uniform. Indeed, there are pilots whose basic profession is close to the drones' use in the company (e.g., inspection) and others whose basic profession is more distant (e.g., drafters). In the second case, this adds a new function to the job and modifies the work more greatly. In particular, the combination of the two functions can lead to a task conflict like RPA, since it can sometimes be difficult to meet both the requirements during a period of high workload. Thus, in the first case the pilots use drones for their daily activities, while in the second they respond to requests from other teams and work for them. This involves studying requests, meeting deadlines and managing the flow of requests to meet all of them.

Drones differ from RPA by allowing pilots to take decisions. Indeed, drones help pilots to consider a variety of information during the flight that they can exploit for selecting 
an action and adapting their behaviors. Conversely, RPA, as an arresting technology, does not permit users to interfere, stop or circumvent the automatic execution of its actions.

\section{The modifications induced on work}

\section{Task characteristics}

In the case of RPA, the technological performance of tasks (e.g., cost search, invoice creation, transfers) has changed the participants' perception of autonomy. In particular, for cost research, the robot offers complete information about the project. Before the robot, this research and cost analysis was not carried out for all projects due to a lack of time, which is why employees had to rely on the information provided by the project manager. Asking questions was quite limited but now they can point out inconsistencies in the project. With all the information, participants feel more in control. Therefore, arresting technology seems to be linked differently as expected to the perception of autonomy.

Concerning drones, in the first case the pilots use the drone to enhance their task performance. Using drones will depend on several elements (i.e. type of asset, accessibility of the asset, mission) but the pilot is completely autonomous in the decision to use it. For inspection purposes, the drone decreases the cumbersome administrative procedures that employees had to go through. The employees put certain lines out of service less often in order to carry out the inspection and are therefore freer to plan them. In the case of missions, pilots are very autonomous in work scheduling: they decide their own schedule and this allows them to manage emergencies or deadlines. They have a great deal of freedom in that they do not have to justify their actions to a supervisor, they only have an outcome accountability where they are expected to deliver results. In both cases, this increases autonomy and can also be partly explained by the fact that few other organization members possess this skill. 
"As a pilot, we have our own schedule, our own program, so we organize ourselves a little bit as we want, so we don't have a leader who says, "Look out, where are you at?" We have deadlines to meet, now we organize our work as we want”. (Participant 5, user) "if now I am asked for a mission... in three weeks, I am free, I plan my mission when I want as long as they receive the results in three weeks". (Participant 6, user).

"I'm much more autonomous for that part anyway. There are only two of us out of the whole service to use it, and therefore, since all the others might not have this skill, we don't ask you any questions, you're free to program it as you wish". (Participant 7, user)

On the other hand, the drone is modifying certain tasks. Before the drone, inspection was done using binoculars, cameras and manual measurements, whereas now it digitizes the information collected. For example, it can provide new data such as an aerial map by reconstructing the area flown over at "perfect scale". In the second case, this leads to new and totally different tasks for the pilots, such as image processing, mission preparation, report creation or risk analysis. In both cases, respondents report more task variety.

Finally, inspection is a rather divided job. Employees inspect and then let others do the works or renovations that need to be done. They never see the end result of their work, just like drafters who do not see the results of their drawings. Their work thus seems to have a weak job identity. Here, in both cases respondents feel that they are accomplishing the tasks a bit more in their totality, enhancing the job identity.

"Yes, I feel more useful, because before ... you draw a plan, you finish the plan, you don't really see. When we draw a plan, we give it to our boss and we get a next plan but we never see..., I never saw after the work site according to the plan I drew” (Participant 6, user) "It's not that we're not in the real world, but we don't see the outcome of our work. We know what it's for, it's super important, but we never see the result in fact" (Participant 7, user). 
While RPA seems to solely modify the level of autonomy, the drone appears to alter other task characteristics that transforms the perceived range of tasks.

\section{Knowledge characteristics}

In regard to RPA, the technology has changed the way of working: previously not all projects were analyzed, and now this is done systematically by the robot. This systematic analysis thus brings more information to the workers and increases the information processing even if workers who cannot process everything. Therefore, the interviewees reported to still rely sometimes on the project manager.

Hence, the division of tasks has also changed between workers and technology. Routine tasks are performed by the robot and more complicated cases - because they cannot be handled by technology - need to be handled by the employees. Moreover, task completion by RPA frees up time for employees that they can spend on managing exceptions, difficult or complex situations, or improving services. Exception management requires the employee to make decisions and find solutions to resolve problems. As the result, jobs in accounting which used to be executive and mainly composed of manual procedures are changing. Henceforth, this new distribution of tasks requires more reflexive work (e.g., problem solving, exception handling) which enhances knowledge characteristics of the job. For instance, when the robot does not work the employee must try to understand why. Specifically for developers, this new role allowed them to acquire new skills. In particular, the developers had to change their way of thinking. If previously their job required more execution, they had to adopt a more abstract way of thinking, such as taking a step back to question existing processes, having a global vision of the process to cut it out or knowing how to transcribe the information collected into code. They also had to write of document for users which seems to have been a less interesting activity for the developers. The acquisition of these skills did not impact their level 
of specialization. Indeed, programming demands a low level of complexity, it requires basic computer skills.

These elements are in line with proposition 2 which assumes that knowledge characteristics may be strengthen by arresting technology. However, this new requirement may not always be well perceived by those who like to perform procedures while for others it is welcomed.

"For me, my day goes faster if I don't have repetitive things to do, if I really have small problems to solve and cases to interpret. I understand that if there are people who like to come to work and like to do standardised stuff, not having too many exceptions can be annoying for them”. (Participant 2, user)

Regarding knowledge characteristics, the drone modifies the image processing. This processing is facilitated by software that automatically analyzes pictures and information (e.g., angle, location, etc.). For example, when a crack on an asset is detected, the software can make a 3D model of it and insert it on the plan, whereas before it had to be redrawn by hand. In information processing, this provides a chronology of inspections which helps to see the evolution of the asset over time and to compare it at different times. Also, the drone and the software seem to reduce information processing. However, piloting of drones brings greater cognitive demands. For example, the flight requires a great deal of mission preparation (e.g., flight authorization, risk analysis), concentration during the flight to take into account obstacles, photo quality or distance appraisal. Nevertheless, over time, a habituation effect sets in as employees often do the same types of missions. Similarly, the complexity of the tasks depends on the missions they perform. Some are a little more complex than others, but this complexity will undoubtedly decrease with habituation. It is therefore likely that these cognitive demands will decrease over time. 
"It depends a little bit on the type of mission because in the long run it becomes a bit of a routine as we start to know our lines since we always do the same regions" (Participant 6, user).

Respondents state that drones bring a demand for constant learning (Xie et al., 2018) as pilots must keep up with new sensors, new drone applications, etc. They discuss among themselves the possible organizational applications and need to keep flight skills and knowledge up to date. Although the authors classify this characteristic out of the other categories, we brought it closer in our analysis of the work cognitive demands and thus knowledge characteristics.

"[...] this technology, what they're coming out with as a product today, is actually outdated because it's like PCs, they've found something else, so you always have to keep up with the times and see what's changing ..." (Participant 7, user).

The pilots have developed a lot of knowledge, both technical (on the mastery of drones and software), theoretical (to pass the pilot's license) and business (on assets). In particular, the drafters had to develop knowledge on the different jobs and specialities of the company in order to take good photos and videos and to complete the missions. Today, they have a greater knowledge of the jobs within the organization because they are in contact with different teams (i.e., clients). Finally, the drone also requires rigour because its use involves some risks even if the piloting itself is not very complicated. In both cases, piloting drones has enabled employees to diversify their skills. The legislative aspects (such as the pilot's licence) and technical aspects increase employee level of specialization, even if the final decision always lies with the client teams who are the experts in their field.

Even if no propositions have been made for drone, this technology seems also to enhance knowledge characteristics, especially for those who were not familiar with inspection in the first place. When comparing results for the two technologies, some specificities appear: 
RPA augments the perception of problem solving while drone intensify the demand for constant learning. Furthermore, some effects can be the result of implementation choices because the development of skill and specialization is observed only for developers (and not RPA users) and pilots who were not familiar with inspection.

\section{Social characteristics}

While no modifications have been observed for RPA users, the programming activity has enabled developers to create relationships outside and within the department. Indeed, in the service there is little interdependence between members and this project has helped to develop a team dynamic. The robot's implementation required exchanges, meetings, coordination of actions and thus created interdependence between members. On the other hand, this project allowed them to be in contact with people outside the department (e.g., IT department) who helped them develop the robot, and this required a mutual understanding of these different teams for the success of the project. To conclude, this development project has increased the social characteristics at work for the developers. Yet, as our proposition 3 concerns users and not developers, the verbatim give no clue about some perceived changes in social characteristics.

For drones, at the relational level little change is reported with colleagues except for jealousies. Some colleagues see only the recreational aspect of the drone and not the instrumental aspect.

"For people who don't pilot, when they see us leaving with the drone for them it's "you're going to have fun". Yes, it's fun, but it's work too, it's not fun" (Participant 7, user).

In the second case, drafters report that they have developed more relationships both outside the organization (e.g., sales, authority, engineers) and within the organization by providing services to other teams. By being more connected, they also get more feedback from others (i.e., positive or negative). In addition, all pilots are part of a community and exchange around 
their practices and feedback. With the superior, pilots from the first case note a little more interest in their work and for both case they have managed to build a trust-based relationship because they bring results. Thus, in the first case, social characteristics are little modified with the introduction of the drone (i.e., manager interest, jealousies) whereas in the second case, social characteristics highly increase (i.e., relationships outside the organization, feedback from others, manager interest, jealousies).

Modifications of social characteristics may also depend on implementation choices. For developers, the social characteristics are enhanced because of the development project and the effects for pilots seem to be a consequence of working for other teams.

\section{Work context characteristics}

Whereas no verbatim concerning those elements have been reported for RPA, it seems that some modifications have occurred for drone because it brings less constraints for the inspection tasks. Indeed, the inspection can sometimes require the use of large resources when areas are difficult to access (e.g., line deactivation, bucket trucks) or when it is necessary to work at night to not interfere with the transport flow during the day. The drone allows easy access to these areas by flying over them and thus requires less equipment to carry out inspections. In addition, it provides information that enables the pilot to assess the relevance of climbing on the asset. The use of the drone therefore implies safer work and for the employees, fewer risks and less physical work. As well, drafters' work is rather sedentary: they work at their desk and are not required to go into the field. Working with the drone allows them to get out which has a positive impact for them.

"As drafters, we are always in front of screens all day long. So with the drone, we have a chance to get out of the office, to not always be in front of the screens so we can get some fresh air". (Participant 5, user) 
It seems that diminishing physical demands is a positive aspect of drones. However, when having a sedentary job, bringing some physical activities is also seen positively. Summary

Regarding RPA, the verbatim give some evidence that its modifications on WC depend on whether or not workers have been involved in RPA development. Moreover, the degree of routine in job can also be a moderating factor in the modifications of arresting technology on work. Indeed, when the degree of routine is high, tasks are likely to be automatable and the more employees see their tasks being automated, the more they can perceive being replaced, thus reducing their perception of autonomy. At the time of study, employees decide whether to use the robot or not in their tasks, so they have full control over the impact of the robot on their work. It may reflect an adaptation period where an old and a new operational mode coexist. When the routine is low, work is not very automatable, so technology will only perform a small part of the human work and it will allow one to save time on daily tasks.

To summarize, some tasks (e.g., information search) are rather perceived as boring, so arresting technology removes the routine, repetitive and non-motivating nature of the task. "It seems less of an effort to get into this task because you feel that the preparatory work is already done so I just have to interpret”. (Participant 2, user)

"Personally, I find it more enjoyable because there's a whole aspect of doing exports, combining numbers in Excel that I don't have to do anymore”. (Participant 2, user) "So far we've only tackled repetitive tasks that aren't very attractive, so I think it's a bit convenient for everyone that there's a robot to do that". (Participant 3, expert) It also allows tasks (e.g., transfers) that were not done systematically or postponed due to lack of time to be performed automatically. These changes are not perceived as profound because for participants the work has not fundamentally changed. 
"It doesn't change anything except that it will be done directly without having to wait".

(Participant 3, expert)

"It's a help but I continue to do my work as I did before". (Participant 2, user)

For drones, the initial job seems to be a moderating factor in the perception of work modifications. In the first case, the job has not fundamentally changed for inspection but only the form. For the second case, it is a completely different job, so it represents a big change, and while the drone implies no modifications on the initial role it also makes it possible to get out of the routine. Moreover, it seems that the way the drone is used (i.e., work for other teams) further impacts the way it changes the employee job by enhancing social relationships and task variety (in doing diverse missions). In short, assisting technology makes it possible to change the routine and motivates employees to develop their skills and knowledge. "All these technologies allow you to reinvest and find other goals and move forward, otherwise it would become routine”. (Participant 7, user).

\section{Relationship with the technology}

Agentic relationship with $R P A$

According to the manager and some participants, because the robot can perform some tasks, some employees feel that they can be replaced by the robot. Nevertheless, the technology is seen by the participants as an aid tool since all tasks cannot be automated with RPA. Participants generally see this conjoined agency with arresting technology as useful. Technology saves employees time in their work by its quick execution so that they can concentrate on other tasks. Indeed, some manual procedures are very time-consuming. They also perceive a gain in terms of quality of work because they have more accurate information. It thus helps workers to achieve higher efficacy at work.

The participants' view of RPA is particularly interesting. The robot is seen as " $a$ virtual team member", "a virtual user", "a colleague" or "a virtual person". It uses the same 
interfaces as employees, so it looks like a remote human colleague working because they can see its work progress. The communication with the robot goes through different channels such as automatic emails from the robot. The employee can also send an email request to it or put a file in its folder. Therefore, the interactions are close to those that one could have with a human (e.g., exchange of emails). In addition, the robot has an avatar, a name and an email address. A contest was held to choose its name. All of this participate for the technology to be perceived differently from other tools. This is an organizational choice in order not to forget the robot and to update it. Regarding proposition 5, it seems that the robot is talked not solely as a tool but as a virtual agent and a team member.

"You tend to consider it a little more like a colleague than a tool that is somewhere running on a server so in the end it's the same thing but the way we positioned it from the beginning because we gave it a name, yes...We also talk about it jokingly that it's our colleague." (Participant 2, user)

"The idea is also that the day the robot is busy twenty-four hours a day, we'll buy a second license, then we'll have to launch a contest for another name, which we don't do at all with other tools." (Participant 2, user)

\section{Non-agentic relationship}

Assisting technology is not seen as a threat. It will not substitute humans because it requires human expertise to be used. It is seen more as a helping tool: "they are my eyes without me having to take any risks" (Participant 7). There is no resistance to using it because it makes work easier. Indeed, employees perceive a usefulness of the technology in the workplace. It improves the accuracy and quality of work and saves time, safety and money for the company. In the case of missions, it helps to solve the problems of other teams with innovative solutions. 
Finally, an employee told us about new work experiences thanks to the association of drones with virtual reality glasses. In particular, the virtual reality glasses bring new sensations and a feeling of immersion and conviviality. This experience is closed to a concept that emerged with digital tools that is user experience (Turner, 2017).

"We even have virtual reality glasses when we pass the drone, we look at it and start moving with it so it's like being a bird, it's funny. We have Google glasses and it's great [...] It's even more friendly to use because the person who is going to look at the defects, it's as if they were immersed, so it's as if they were in a bucket truck and then they start looking for those defects [...] it's nice with the glasses too." (Participant 7, user)

Other elements can be identified in other pilots' speech.

"I thought it was a very beautiful machine, a beautiful tool that could provide good help in terms of safety and punctuality. So the fact that we will always have better performing tools, that we will always be able to increase the precision and the range of distances, it motivates us to learn more and more and to evolve in it and somewhere to increase this performance and try to satisfy as many people as possible". (Participant 6, user).

"Me as a pilot, it's my hobby to do it at work with these technologies because we don't have the same budgets, so we fly with super machines, it's great". (Participant 5, user)

First, the worker seems to have positive affect in the use of the virtual reality glasses ("more friendly", “it's nice”). Second, it appears that the technology has an instrumental value as the virtual reality helps an expert to look at the defects without being temporally with the pilots, in addition drones have also instrumental value ("providing good help with safety and punctuality [...] increasing accuracy”). Third, drones and virtual reality seem related to an aesthetic experience ("beautiful machine”, "it's like being a bird"). Finally, there is some evidence for hedonic aspects in the interaction with the technologies ("it's my hobby", "funny"). Further research will be needed to determine if this finding is specific to virtual 
reality or if this pattern is found with other technologies or other combinations of technologies.

When comparing the two technologies, they both have positive impacts on work (e.g., enhanced quality, time efficiency) attesting of their instrumental value. However, it also seems that technological equipment use is different from non-technological equipment use and that technologies are gaining significance at work. Indeed, our technological use goes beyond the purely instrumental value of equipment: hedonic and conviviality aspects emerge from the interactions with the drone and RPA is considered not only as a tool but as a virtual colleague. These results give some clues that are consistent with proposition 4 .

\section{DISCUSSION}

The purpose of this study was to propose potential modifications on WC after the introduction of digital technologies in the workplace. By introducing a classification of digital technologies, we studied the modifications brought by two out of four types of technology (i.e., arresting, assisting). Our analysis indicated that contrary to our expectations arresting technology may enhance employee autonomy by offering more information to workers. Arresting technology does not seem to curtail the level of autonomy because it accomplishes less interesting tasks that are perceived as cumbersome. With this new division of tasks, participants felt that they were spending more time on tasks with added value. Therefore, the agency of arresting technology (i.e., action selection) may not be seen as a threat for human agency as long as it executes tasks that require low human control and expertise. The new distribution of tasks raises the knowledge characteristics of jobs in accounting as workers carry out more reflective work to handle expertise tasks. This is in line with the results of the Eurofound (2018) study. Finally, despite the lack of propositions for assisting technology, our analysis about drones demonstrated that they have a positive relation with the level of autonomy and knowledge characteristics. 
These findings might be interpreted in the light of Shneiderman's (2020) framework which proposes that human autonomy and technological automation are not necessarily contradictory. Human autonomy and automation are beneficial in different situations but the combination of a high level of human autonomy and a high level of automation are better suited in some situations. For tasks of great expertise, human autonomy should be favoured whereas automation is recommended for well-understood predictable tasks such as simple situations or situations which require quick reactions (e.g., airbags). The conjunction of human and technological control is suggested for complex and difficult tasks where creative decisions are needed. As noted, assisting technologies let the action selection and the protocol development to humans and thus workers control entirely the execution of tasks.

Consequently, they appear more appropriate for tasks of great expertise. Arresting technologies automate standardized tasks that can be broken down into steps thus they seem appropriate for simple tasks that do not require a great human control. This framework could explain why the changes brought by the two digital technologies (i.e., drones, RPA) on employee WC have not been negatively perceived by the participants: first because of the fit between tasks and the application of technology and second because these technologies do not threaten human control. This is presumably why a common theme appeared about technological aid and usefulness.

Our study may have some contributions for the WD model. First, the dimension of equipment use should be refined to distinguish non-technological equipment from technological equipment. As our relationship with technologies are constant and as our channels of communication with technologies look like human interactions, they might be considered apart from other tools and machines. Like Parker and Grote (2020) noted, "the relationship between humans and $\mathrm{AI}$ is fundamentally different from the relationships of humans and technology of the past as both humans and technology have agency". 
Technologies are endowed with agency capabilities (Murray et al., 2020) which suggest that they could be perceived as non-human agents that have complex relationships with human agents. In the scientific literature, this idea has already been developed through the Computer as Social Actors paradigm (Nass et al., 1994) and the Actor Network Theory (Latour, 2005) which considers technology as a non-human actor and suggests studying human and nonhuman actors which are acting at the same level. Besides, in some cases human-technology interactions can be quite similar as human interactions (Broadbent, 2017). Therefore, we propose that as human relationships are a main category of $\mathrm{WC}$, human-technology relationships should be considered as a main category that to some extent might motivate workers. For instance, gamification offers insights into the way technology can motivate employees. Gamification refers to the insertion of principles and features of game into nonplayful environment such as work. It adopts the motivational basis of games with cognitive, emotional and social aspects (Domínguez et al., 2013). In addition, it has been proposed that gamification allows people to reach a state of flow (Brockmyer et al., 2009; Procci et al., 2012) as it is the case with immersive technologies (Suh \& Prophet, 2018).

Besides, the combination of drones and virtual reality devices (i.e., immersive technology) - two technologies that we classified as assisting technologies - seems to produce surprising results. Further research is needed to understand if this new work experience that can be labelled as a user experience (Turner, 2017) is observed solely with immersive technologies. Immersive technologies are designed to maximize pleasure and enjoyment which are usually identified as user experience's components. However, Turner (2017) advocates that user experience encompasses a larger range of experience (e.g., involvement, sense-making processes) and emphasizes the necessity for psychological scholars to extend our knowledge on the human-technology interactions. 
Second, given the growth of knowledge demands and process in jobs, employees are likely to spend more time at their desks working with technological devices. The WD model assess for seating arrangements and posture that will be more relevant as work becomes sedentary. However, as work will develop into more knowledgeable jobs and shifts will occur as for RPA (i.e., decrease of manual procedures), it would be pertinent to take into account the motivational factor of non-sedentary work. Indeed, as a participant mentioned, this shift might not be well received by all workers, especially manual workers whose habits will be highly disrupted. For manual workers, changes in work context characteristics (e.g., less risk of accident, less physical demands) may positively impact employee outcomes up to a certain point where the impact may be reversed as they will miss executing formal procedures.

Furthermore, our study may also contribute to the classification of Murray et al., (2020). First, we submitted a technological classification to help future studies to tackle the process by which digital technologies bring changes in the work processes as advocated by Barley (2015). Because the study of human and managerial aspects has been neglected in the digitalization process of organizations (Oesterreich \& Teuteberg, 2016; Schneider, 2018), this enhanced framework may support future studies to hypothesize their effects for employees. Second, we also offered insight into how the human-nonhuman ensemble is perceived by workers and, this perception differs depending on the type of technology. For instance, it seems that working with an arresting technology bolsters the perception of the technology as a non-human agent as well as a threat for human jobs while working with an assisting technology may generate a motivating collaboration.

\section{Limitations}

Because of our small sample size and a single organizational context, our findings should be taken with caution. Other studies in different organizational contexts and with different methodologies are needed to replicate these findings. This will help to counteract the 
effect of other factors that reflect managerial and organizational choices about the introduction of technologies (e.g., creation of a development team). The second limit is related to our sample size and involves the fact that some types of technologies (i.e., augmenting, automating) have not been analyzed. Therefore, the examination of our propositions is not complete. Our study constitutes a first exploration of the link between digital technologies and $\mathrm{WC}$, so other research (qualitative or quantitative) is necessary to improve our knowledge about it.

A third limitation lies in one fundamental characteristic of digitalization namely system integration. As digital technologies are more integrative, it is more likely that the introduction of one digital technology will be followed by others. As a result, their modifications on WC may be combined like drones and virtual reality or may be greatly different, then our study might have only pointed out trends in future jobs (i.e., an increase in knowledge characteristics).

Finally, researchers may have pointed out several limits to the thematic analysis. Following some guidelines (Braun \& Clarke, 2006), we strove to make our choices and our assumptions explicit and to match our epistemological assumptions with our methodology. We sought to meet their criteria to conduct a good thematic analysis. As a result, we put ourselves in an active position to search themes because they do not just appear, and we tried not to paraphrase what participants have reported. Then, we aimed at providing coherence between our data set and our analysis as well as between our analysis and the WD model.

\section{Future research}

Future research can investigate the new environment created by digital technologies that we called digital work context. Because work context is likely to influence work situations and organizational behaviors in many different ways (Johns, 2006), it is highly relevant to study and detail it. Besides, characterizing the new work context will help the 
scientific community to recognize how the theories and models need to adapt to describe new situations at work. Observational studies will be needed to bring together influences from several digital technologies. These studies have illustrated that technological changes bring opportunities for changes at the relational level, especially in power relations where cooperation or conflicts can emerge (e.g., Barrett et al., 2012; Plesner \& Raviola, 2016). The introduction of new technologies may also come with modifications of the physical space (e.g., Barrett et al., 2012). Such findings will be valuable to gather data about changes in the omnibus context and its new features in order to grasp its effect on organizational behaviors, attitudes and cognitions.

Other studies can investigate the other types of technologies to gather knowledge about the modifications they induce on the $\mathrm{WC}$ and maybe identify a common pattern in the changes. These studies can be qualitative or quantitative although conducting quantitative studies may confirm the results presented here. To expand these findings, further quantitative studies may integrate other factors that have an important role in organizational changes or WC such as manager behaviors (Bareil, 2009; Parker, Van den Broeck, et al., 2017). Indeed, managers play a critical role in shaping one's perception of a change (e.g., introduction of a technology) by the resources they offer (e.g., information, training), by their relationship with the employees or their leadership. For instance, our findings seem to show that new technologies may enhance both the level of autonomy and cognitive demands. According to the job demands-resources model (Demerouti et al., 2001), employee well-being may thus not be impaired. Therefore, managerial choices taken regarding the introduction of new technologies are important to prevent negative consequences such as stress or workload. As the social information processing theory states (Salancik \& Pfeffer, 1978), it is possible that social cues like the perceived transparency of the change, the relationship between the manager and the employee or the leadership shape the perceived modifications on the WC. 
Likewise, quantitative studies may develop measures to operationalize the concept highlighted here, namely the perception of the technology as a non-human agent. Further research unravelling the multiple dimensions in the human-technology relationships are also needed. These studies may adopt a mix design (i.e., qualitative and quantitative) to explore and reveal the important features nested in these relations and to measure and validate them.

Longitudinal studies will also be helpful to capture the perceptions of one's WC before the technological change and track these perceptions after the changes at different times to compare them. Therefore, it will be possible to untangle the persistent effects and the transient effects (e.g., habituation effect) on work design and employee outcomes (e.g., job satisfaction) but also to distinguish primary and early effects from latter effects. For example, Barley (1990) suggested that technology may bring changes firstly into the nonrelational elements of work role (e.g., tasks, skills) and secondly into the relational elements of work role. However, acknowledging that today technological changes are constant such design might be difficult to carry out.

Moreover, future research might be interested to further extend our knowledge about the demand for constant learning that is a new work characteristic developed by Xie et al., (2018). The demand for constant learning has been investigated under the job demand-control framework and mixed effects have been found on employee attitudes (e.g., job satisfaction, occupational commitment). This dimension has the highest correlations with motivational characteristics (comprising both task-related and knowledge-related dimensions), hence further studies are required to better understand the empirical links between them. The authors have acknowledged that demand for constant learning may participate to the individual growth through knowledge and skill acquisition, therefore according to Morgeson and Humphrey (2006) definition this dimension might be insert into knowledge characteristics. 


\section{References}

Ackerman, P. L., \& Kanfer, R. (2020). Work in the 21st Century : New Directions for Aging and Adult Development. American Psychologist, 75(4), 486-498. https://doi.org/10.1037/amp0000615

Ashford, S. J., De Stobbeleir, K. E., \& Nujella, M. (2016). To Seek or Not to Seek: Is that the Only Question? Recent Developments in Feedback-Seeking Literature. Annual Review of Organizational Psychology and Organizational Behavior, 1-27. https://doi.org/10.1146/annurev-orgpsych-041015-062314

Bandura, A. (1982). Self-efficacy mechanism in human agency. American Psychologist, 37(2), 122-147. https://doi.org/10.1037/0003-066X.37.2.122

Bandura, A. (1997). Self-Efficacy: the exercise of control. Freeman.

Bandura, A. (2008). Reconstrual of "Free Will" From the Agentic Perspective of Social Cognitive Theory. In J. Baer, J. C. Kaufman, \& R. F. Baumeister (Eds.), Are We Free? Psychology and Free Will (pp. 1689-1699). Oxford University Press. https://doi.org/10.1017/CBO9781107415324.004

Bareil, C. (2009). Gérer le volet humain du changement (Managing the human side of change). Les Editions Transcontinental.

Barley, S. R. (2015). Why the internet makes buying a car less loathsome: How technologies change role relations. Academy of Management Discoveries, 1(1), 31-60. https://doi.org/10.5465/amd.2013.0016

Battistelli, A., \& Odoardi, C. (2018). Les défis de la 4ème révolution industrielle : la dynamique entre le changement et l'innovation (The challenges of the 4th industrial revolution: the dynamic between change and innovation). In M. Lauzier \& N. Lemieux (Eds.), Améliorer la gestion du changement dans les organisations (Improve change management in organizations). Presses de l'Université du Québec.

Braun, V., \& Clarke, V. (2006). Using thematic analysis in psychology. Qualitative Research in Psychology, 3, 77-101. 10.1191/1478088706qp063oa

Brenon, A., Portet, F., \& Vacher, M. (2016). Preliminary study of adaptive decision-making system for vocal command in smart home. 12th International Conference on Intelligent Environments (IE) (pp. 218-221). 10.1109/IE.2016.49

Brettel, M., Friederichsen, N., Keller, M., \& Rosenberg, M. (2014). How Virtualization, Decentralization and Network Building Change the Manufacturing Landscape: An Industry 4.0 Perspective. International Journal of Information and Communication 
Engineering, 8(1), 37-44. https://doi.org/10.5281/zenodo.2658202

Broadbent, E. (2017). Interactions with Robots: The Truths We Reveal About Ourselves. Annual Review of Organizational Psychology and Organizational Behavior, 68, 627652. https://doi.org/10.1146/annurev-psych-010416-043958

Brockmyer, J. H., Fox, C. M., Curtiss, K. A., McBroom, E., Burkhart, K. M., \& Pidruzny, J. N. (2009). The development of the Game Engagement Questionnaire: A measure of engagement in video game-playing. Journal of Experimental Social Psychology, 45(4), 624-634. https://doi.org/10.1016/j.jesp.2009.02.016

Brynjolfsson, E., \& McAfee, A. (2014). The second machine age: Work, progress, and prosperity in a time of brilliant technologies. WW Norton \& Company.

Campion, M. A. (1988). Interdisciplinary Approaches to Job Design: A Replication with Methodological Extensions. Journal of Applied Psychology, 73(3), 467-481. https://doi.org/10.5465/ambpp.1987.17534233

Cascio, W. F., \& Montealegre, J. R. (2016). How Technology is Changing Work and Organizations. Annual Review of Organizational Psychology and Organizational Behavior, 3, 349-375. https://doi.org/10.1146/annurev-orgpsych-041015-062352

Davidsson, P., Hajinasab, B., Holmgren, J., Jevinger, Å., \& Persson, J. A. (2016). The fourth wave of digitalization and public transport: Opportunities and challenges. Sustainability, 8(12), 1248. https://doi.org/10.3390/su8121248

Deci, E. L., \& Ryan, R. M. (1985). Intrinsic Motivation and Self-Determination in Human Behavior. Plenum.

Demerouti, E., Bakker, A. B., Nachreiner, F., \& Schaufeli, W. B. (2001). The Job DemandsResources Model of Burnout. Journal of Applied Psychology, 86(3), 499-512. https://doi.org/10.1037/0021-9010.86.3.499

Deng, X., \& Joshi, K. D. (2016). Why Individuals Participate in Micro-task Crowdsourcing Work Environment: Revealing Crowdworkers' Perceptions. Journal of the Association for Information Systems, 17(10), 648-673. https://doi.org/10.17705/1jais.00441

Díaz, R. I., \& Cabrera, D. D. (1997). Safety climate and attitude as evaluation measures of organizational safety. Accident Analysis \& Prevention, 29(5), 643-650. https://doi.org/10.1016/S0001-4575(97)00015-8

Domínguez, A., Saenz-De-Navarrete, J., De-Marcos, L., Fernández-Sanz, L., Pagés, C., \& Martínez-Herráiz, J. J. (2013). Gamifying learning experiences: Practical implications and outcomes. Computers \& Education, 63, 380-392.

https://doi.org/10.1016/j.compedu.2012.12.020 
Eriksson-Zetterquist, U., Lindberg, K., \& Styhre, A. (2009). When the good times are over: Professionals encountering new technology. Human relations, 62(8), 1145-1170. https://doi.org/10.1177/0018726709334879

Eurofound. (2018). New tasks in old jobs: Drivers of change and implications for job quality. Publications Office of the European Union. http://eurofound.link/fomeef18004

Feldman, M. S. (2000). Organizational routines as a source of continuous change. Organization Science, 11(6), 611-629. https://doi.org/10.1287/orsc.11.6.611.12529

Fiore, S. M., Wiltshire, T. J., Lobato, E. J., Jentsch, F. G., Huang, W. H., \& Axelrod, B. (2013). Toward understanding social cues and signals in human-robot interaction: effects of robot gaze and proxemic behavior. Frontiers in psychology, 4, 859. https://doi.org/10.3389/fpsyg.2013.00859

Flin, R., Mearns, K., O'Connor, P., \& Bryden, R. (2000). Measuring safety climate: identifying the common features. Safety science, 34(1-3), 177-192. https://doi.org/10.1016/S0925-7535(00)00012-6

Frey, C. B., \& Osborne, M. A. (2013). The future of employment: How susceptible are jobs to computerisation. University of Oxford

Fried, Y. (1991). Meta-Analytic Comparison of the Job Diagnostic Survey and Job Characteristics Inventory as Correlates of Work Satisfaction and Performance. Journal of Applied Psychology, 76(5), 690-697. https://doi.org/10.1037/0021-9010.76.5.690

Gibson, C. B., Gibbs, J. L., Stanko, T. L., Tesluk, P., \& Cohen, S. G. (2011). Including the "I" in Virtuality and Modern Job Design: Extending the Job Characteristics Model to Include the Moderating Effect of Individual Experiences of Electronic Dependence and Copresence. Organization Science, 22(6), 1481-1499. https://doi.org/10.1287/orsc.1100.0586

Grant, A. M., Fried, Y., Parker, S. K., \& Frese, M. (2010). Putting job design in context: Introduction to the special issue. Journal of Organizational Behavior, 31, 145-157. https://doi.org/10.1002/job.679

Hackman, J. R., \& Oldham, G. R. (1975). Development of the Job Diagnostic Survey. Journal of Applied Psychology, 60(2), 159-170. https://doi.org/10.1037/h0076546

Hermann, M., Pentek, T., \& Otto, B. (2016). Design principles for industrie 4.0 scenarios. System Sciences (HICSS), 49th Hawaii International Conference (pp. 3928-3937). 10.1109/HICSS.2016.488

Hofmann, E., \& Rüsch, M. (2017). Industry 4.0 and the current status as well as future prospects on logistics. Computers in Industry, 89, 23-34. 


\section{https://doi.org/10.1016/j.compind.2017.04.002}

Howard, A. (1995). The changing nature of work. Jossey-Bass

Howitt, D. (2016). Introduction to qualitative research methods in psychology: Putting theory into practice ( $3^{\text {rd }}$ edition). Pearson.

Humphrey, S. E., Nahrgang, J. D., \& Morgeson, F. P. (2007). Integrating Motivational, Social, and Contextual Work Design Features: A Meta-Analytic Summary and Theoretical Extension of the Work Design Literature. Journal of Applied Psychology, 92(5), 1332-1356. https://doi.org/10.1037/0021-9010.92.5.1332

Inkermann, D., Schneider, D., Martin, N. L., Lembeck, H., Zhang, J., \& Thiede, S. (2019). A framework to classify Industry 4.0 technologies across production and product development. Procedia CIRP, 84, 973-978. https://doi.org/10.1016/j.procir.2019.04.218

Irizarry, J., Gheisari, M., \& Walker, B. N. (2012). Usability assessment of drone technology as safety inspection tools. Journal of Information Technology in Construction (ITcon), 17(12), 194-212. https://www.itcon.org/2012/10

Jetter, J., Eimecke, J., \& Rese, A. (2018). Augmented reality tools for industrial applications: What are potential key performance indicators and who benefits? Computers in Human Behavior, 87, 18-33. https://doi.org/10.1016/j.chb.2018.04.054

Jiang, F., Jiang, Y., Zhi, H., Dong, Y., Li, H., Ma, S., ... \& Wang, Y. (2017). Artificial intelligence in healthcare: past, present and future. Stroke and vascular neurology, 2(4), 230-243. http://dx.doi.org/10.1136/svn-2017-000101

Johns, G. (2010). Some unintended consequences of job design. Journal of Organizational Behavior, 31, 361-369. https://doi.org/10.1002/job.669

Karasek, R. A. (1979). Job Demands, Job Decision Latitude, and Mental Strain: Implications for Job Redesign. Administrative Science Quarterly, 24. doi: 10.2307/2392498

Kemp, N. J., \& Clegg, C. W. (1987). Information technology and job design: A case study on computerized numerically controlled machine tool working. Behaviour and Information Technology, 6(2), 109-124. https://doi.org/10.1080/01449298708901821

Kipper, L. M., Furstenau, L. B., Hoppe, D., Frozza, R., \& Iespen, S. (2019). Scopus scientific mapping production in industry 4.0 (2011-2018): a bibliometric analysis. International Journal of Production Research. https://doi.org/10.1080/00207543.2019.1671625

Koceski, S., \& Koceska, N. (2016). Evaluation of an assistive telepresence robot for elderly healthcare. Journal of medical systems, 40(5), 121. https://doi.org/10.1007/s10916-0160481-X

Kohler, D. \& Weisz, J. (2016). Industrie 4.0 : comment caractériser cette quatrième révolution 
industrielle et ses enjeux ? (Industry 4.0: How can we characterize this fourth industrial revolution and its challenges?). Annales des Mines - Réalités industrielles, 4, 51-56. https://doi.org/10.3917/rindu1.164.0051

Latour, B. (2005). Reassembling the Social: An introduction to actor-network-theory. Oxford University Press

Lee, M. K. (2018). Understanding perception of algorithmic decisions: Fairness, trust, and emotion in response to algorithmic management. Big Data \& Society, 5(1). https://doi.org/10.1177/2053951718756684

Lughofer, E., \& Sayed-Mouchaweh, M. (2019). Prologue: Predictive Maintenance in Dynamic Systems. In E. Lughofer \& M. Sayed-Mouchaweh (Eds), Predictive Maintenance in Dynamic Systems (pp.1-23). Springer, Cham

Lupushor, S., \& Fradera, A. (2017). The Future of Work. In G. Hertel, D. L. Stone, R. D. Johnson \& J. Passmore (Eds), The Wiley Blackwell Handbook of the Psychology of the Internet at Work (pp. 481-508). John Wiley \& Sons

McFarland, L. A., \& Ployhart, R. E. (2015). Social media: A contextual framework to guide research and practice. Journal of Applied Psychology, 100(6), 1653-1677. https://doi.org/10.1037/a0039244

Mendling, J., Decker, G., Hull, R., Reijers, H. A., \& Weber, I. (2018). How do Machine Learning, Robotic Process Automation, and Blockchains Affect the Human Factor in Business Process Management? Communications of the Association for Information Systems, 43, 1-23. 10.17705/1CAIS.04319

Morgeson, F. P., \& Campion, M. A. (2002). Minimizing tradeoffs when redesigning work: Evidence from a longitudinal quasi-experiment. Personnel Psychology, 55, 5889-612. https://doi.org/10.1111/j.1744-6570.2002.tb00122.x

Morgeson, F. P., \& Humphrey, S. E. (2006). The Work Design Questionnaire (WDQ):

Developing and validating a comprehensive measure for assessing job design and the nature of work. Journal of Applied Psychology, 91(6), 1321-1339.

\section{https://doi.org/10.1037/0021-9010.91.6.1321}

Murray, A., Rhymer, J., \& Sirmon, D. (2020). Humans and technology: Forms of conjoined agency in organizations. Academy of Management Review.

https://doi.org/10.5465/amr.2019.0186

Nass, C., Steuer, J., \& Tauber, E. R. (1994). Computers are social actors. Proceedings of the SIGCHI conference on Human factors in computing systems (pp 72-78).

\section{https://doi.org/10.1145/191666.191703}


O’Kane, P., Smith, A., \& Lerman, M. P. (2019). Building Transparency and Trustworthiness in Inductive Research Through Computer-Aided Qualitative Data Analysis Software. Organizational Research Methods. https://doi.org/10.1177/1094428119865016

Oesterreich, T. D., \& Teuteberg, F. (2016). Understanding the implications of digitisation and automation in the context of Industry 4.0: A triangulation approach and elements of a research agenda for the construction industry. Computers in Industry, 83, 121-139. https://doi.org/10.1016/j.compind.2016.09.006

Oh, C., Lee, T., Kim, Y., Park, S., Kwon, S., \& Suh, B. (2017). Us vs. Them: Understanding Artificial Intelligence Technophobia over the Google DeepMind Challenge Match. Proceedings of the CHI Conference on Human Factors in Computing Systems (pp. 25232534). https://doi.org/10.1145/3025453.3025539

Oldham, G. R., \& Hackman, J. R. (2010). Not what it was and not what it will be: The future of job design research. Journal of Organizational Behavior, 31, 463-479. https://doi.org/10.1002/job.678

Oztemel, E., \& Gursev, S. (2018). Literature review of Industry 4.0 and related technologies. Journal of Intelligent Manufacturing, 31, 127-182. https://doi.org/10.1007/s10845-018$\underline{1433-8}$

Parasuraman, R., \& Manzey, D. H. (2010). Complacency and Bias in Human Use of Automation: An Attentional Integration. Human Factors, 52(3), 381-410.

\section{https://doi.org/10.1177/0018720810376055}

Parker, S. K. (2014). Beyond Motivation: Job and Work Design for Development, Health, Ambidexterity, and More. Annual Review of Organizational Psychology and Organizational Behavior, 65, 661-691. https://doi.org/10.1146/annurev-psych-010213$\underline{115208}$

Parker, S. K., \& Grote, G. (2020). Automation, Algorithms, and Beyond: Why Work Design Matters More Than Ever in A Digital World. Applied Psychology.

\section{https://doi.org/10.1111/apps.12241}

Parker, S. K., Morgeson, F. P., \& Johns, G. (2017). One Hundred Years of Work Design Research: Looking Back and Looking Forward. Journal of Applied Psychology, 102(3), 403-420. https://doi.org/10.1037/ap10000106

Parker, S. K., Van den Broeck, A., \& Holman, D. J. (2017). Work Design Influences: A Synthesis of Multilevel Factors that Affect the Design of Jobs. Academy of Management Annals, 11(1), 267-308. https://doi.org/10.5465/annals.2014.0054

Parker, S. K., \& Wall, T. D. (1998). Modern Manufacturing and the Work Design Agenda. In 
S. K. Parker \& T. D. Wall (Eds), Job and Work Design: Organizing Work to Promote Well-Being and Effectiveness (pp. 57-84). SAGE Publications, Inc.

Petrillo, A., De Felice, F., Cioffi, R., \& Zomparelli, F. (2018). Fourth Industrial Revolution: Current Practices, Challenges, and Opportunities. In A. Petrillo, R. Cioffi \& F. De Felice (Eds), Digital Transformation in Smart Manufacturing (pp. 1-20). https://doi.org/10.5772/intechopen.72304

Plesner, U., \& Raviola, E. (2016). Digital technologies and a changing profession: New management devices, practices and power relations in news work. Journal of Organizational Change Management, 29(7), 1044-1065. https://doi.org/10.1108/JOCM09-2015-0159

Powles, J., Hodson, H. (2017). Google DeepMind and healthcare in an age of algorithms. Health Technol. 7, 351-367. https://doi.org/10.1007/s12553-017-0179-1

Pozdnyakova, U. A., Golikov, V. V., Peters, I. A., \& Morozova, I. A. (2019). Genesis of the revolutionary transition to industry 4.0 in the 21 st century and overview of previous industrial revolutions. In E. Popkova, Y. Ragulina \& A. Bogoviz (Eds), Industry 4.0: Industrial Revolution of the 21st Century (pp. 11-19). Springer, Cham

Procci, K., Singer, A. R., Levy, K. R., \& Bowers, C. (2012). Measuring the flow experience of gamers: An evaluation of the DFS-2. Computers in Human Behavior, 28(6), 23062312. https://doi.org/10.1016/j.chb.2012.06.039

Richter, A., Heinrich, P., Stocker, A., \& Schwabe, G. (2018). Digital Work Design: The Interplay of Human and Computer in Future Work Practices as an Interdisciplinary (Grand) Challenge. Business and Information Systems Engineering, 60(3), 259-264. https://doi.org/10.1007/s12599-018-0534-4

Rubin, V. L., Chen, Y., \& Thorimbert, L. M. (2010). Artificially intelligent conversational agents in libraries. Library Hi Tech, 28(4), 496-522. https://doi.org/10.1108/07378831011096196

Salancik, G. R., \& Pfeffer, J. (1978). A social information processing approach to job attitudes and task design. Administrative Science Quarterly, 23(2), 224-253. doi: $10.2307 / 2392563$

Salkin, C., Oner, M., Ustundag, A., \& Cevikcan, E. (2018). A Conceptual Framework for Industry 4.0. In Industry 4.0: Managing the Digital Transformation (pp. 3-23). Springer, Cham. https://doi.org/10.1007/978-3-319-57870-5 1

Saunders, C., Wiener, M., Klett, S., \& Sprenger, S. (2017). The impact of mental representations on ICT-related overload in the use of mobile phones. Journal of 
Management Information Systems, 34(3), 803-825.

https://doi.org/10.1080/07421222.2017.1373010

Schneider, P. (2018). Managerial challenges of Industry 4.0: an empirically backed research agenda for a nascent field. Review of Managerial Science, 12(3), 803-848.

https://doi.org/10.1007/s11846-018-0283-2

Schroeder, A. N., Bricka, T. M., \& Whitaker, J. H. (2019). Work design in a digitized gig economy. Human Resource Management Review.

https://doi.org/10.1016/j.hrmr.2019.100692

Schuh, G., Anderl, R., Gausemeier, J., ten Hompel, M., \& Wahlster, W. (2017). Industry 4.0 Maturity Index. Managing the Digital Transformation of Companies. Acatech - National Academy of Science and Engineering.

Schwab, K. (2017). La quatrième révolution industrielle (The Fourth Industrial Revolution). Dunod.

Schwarzmüller, T., Brosi, P., Duman, D., \& Welpe, I. M. (2018). How Does the Digital Transformation Affect Organizations? Key Themes of Change in Work Design and Leadership. Management Revue, 29(2), 114-138. https://doi.org/10.5771/0935-9915$\underline{2018-2-114}$

Seligman, M. E. (1972). Learned helplessness. Annual review of medicine, 23(1), 407-412.

Sewell, G., Barker, J. R., \& Nyberg, D. (2012). Working under intensive surveillance: When does 'measuring everything that moves' become intolerable? Human Relations, 65(2), 189-215. https://doi.org/10.1177/0018726711428958

Shneiderman, B. (2020). Human-Centered Artificial Intelligence: Reliable, Safe \& Trustworthy. International Journal of Human-Computer Interaction, 36(6), 495-504. https://doi.org/10.1080/10447318.2020.1741118

Skitka, L. J., Mosier, K., \& Burdick, M. D. (2000). Accountability and automation bias. International Journal of Human Computer Studies, 52(4), 701-717. https://doi.org/10.1006/ijhc.1999.0349

Spada, S., Ghibaudo, L., Gilotta, S., Gastaldi, L., \& Cavatorta, M. P. (2017). Analysis of exoskeleton introduction in industrial reality: main issues and EAWS risk assessment. In R. Goonetilleke \& W. Karwowski (Eds), Advances in Intelligent Systems and Computing (pp. 236-244). Springer, Cham. https://doi.org/10.1007/978-3-319-60825-9 26

Suh, A., \& Prophet, J. (2018). The state of immersive technology research: A literature analysis. Computers in Human Behavior, 86, 77-90. https://doi.org/10.1016/j.chb.2018.04.019 
Trist, E. L., \& Bamforth, K. W. (1951). Some Social and Psychological Consequences of the Longwall Method of Coal-Getting: An Examination of the Psychological Situation and Defences of a Work Group in Relation to the Social Structure and Technological Content of the Work System. Human Relations, 4(1), 3-38.

https://doi.org/10.1177/001872675100400101

Turner, P. (2017). A Psychology of User Experience. Springer International Publishing. https://doi.org/10.1007/978-3-319-70653-5

Wall, T. D., Corbett, J. M., Clegg, C. W., Jackson, P. R., \& Martin, R. (1990). Advanced manufacturing technology and work design: Towards a theoretical framework. Journal of Organizational Behavior, 11(3), 201-219. https://doi.org/10.1002/job.4030110304

Wang, B., Liu, Y., \& Parker, S. K. (2020). How does the use of information communication technology affects individuals: A work design perspective. Academy of Management Annals. https://doi.org/10.5465/annals.2018.0127

Waschull, S., Bokhorst, J. A. C., Molleman, E., \& Wortmann, J. C. (2019). Work design in future industrial production: Transforming towards cyber-physical systems. Computers and Industrial Engineering, 139. https://doi.org/10.1016/j.cie.2019.01.053

Weller, C., Kleer, R., \& Piller, F. T. (2015). Economic implications of 3D printing: Market structure models in light of additive manufacturing revisited. International Journal of Production Economics, 164, 43-56. https://doi.org/10.1016/j.ijpe.2015.02.020

Wilkesmann, M., \& Wilkesmann, U. (2018). Industry 4 . 0 - Organizing Routines or Innovations? Journal of Information and Knowledge Management Systems, 48(2), 238254. https://doi.org/10.1108/VJIKMS-04-2017-0019

Wood, R. E. (1986). Task complexity: Definition of the construct. Organizational behavior and human decision processes, 37(1), 60-82. https://doi.org/10.1016/07495978(86)90044-0

Xie, J. L., Elangovan, A. R., Hu, J., \& Hrabluik, C. (2018). Charting New Terrain in Work Design: A Study of Hybrid Work Characteristics. Applied Psychology, 68(3), 479-512. https://doi.org/10.1111/apps.12169

Yoo, Y., Lyytinen, K. J., Boland, R. J., Berente, N., Gaskin, J., Schutz, D., \& Srinivasan, N. (2010). The Next Wave of Digital Innovation: Opportunities and Challenges: A Report on the Research Workshop 'Digital Challenges in Innovation Research'. SSRN Electronic Journal. https://doi.org/10.2139/ssrn.1622170

Zammuto, R. F., Griffith, T. L., Majchrzak, A., Dougherty, D. J., \& Faraj, S. (2007). Information Technology and the Changing Fabric of Organization. Organization 
Science, 18(5), 749-762. https://doi.org/10.1287/orsc.1070.0307

Zuboff, S. (1988). In the Age of the Smart Machine: The Future of Work and Power.

Heinemann professional publishing.

Table 1.

Summary table about the potential effect of each type of technology on the WC

\begin{tabular}{|c|c|c|c|c|}
\hline \multirow{2}{*}{$\begin{array}{l}\text { Types of } \\
\text { technology }\end{array}$} & \multicolumn{4}{|c|}{ Work Characteristics } \\
\hline & Autonomy & $\begin{array}{c}\text { Knowledge } \\
\text { characteristics }\end{array}$ & Social characteristics & $\begin{array}{l}\text { Work context } \\
\text { characteristics }\end{array}$ \\
\hline $\begin{array}{l}\text { Arresting } \\
\text { technology }\end{array}$ & $\begin{array}{l}\text { Modification due to a } \\
\text { decrease of } \\
\text { involvement in tasks } \\
\text { and a bias favouring }\end{array}$ & $\begin{array}{l}\text { Modification due to the } \\
\text { redistribution of tasks. } \\
\text { Human exception } \\
\text { handling may enhance } \\
\text { perception of these } \\
\text { characteristics }\end{array}$ & $\begin{array}{l}\text { Modification due to less } \\
\text { human intermediates } \\
\text { for task execution }\end{array}$ & \multirow{4}{*}{$\begin{array}{l}\text { Modification in } \\
\text { equipment use due } \\
\text { to the increasing } \\
\text { importance of } \\
\text { quality of } \\
\text { equipment and } \\
\text { technology }\end{array}$} \\
\hline $\begin{array}{l}\text { Augmenting } \\
\text { technology }\end{array}$ & $\begin{array}{l}\text { technological } \\
\text { judgment }\end{array}$ & $\begin{array}{l}\text { Modification due to a } \\
\text { reduction of } \\
\text { opportunities to develop } \\
\text { or reinforce self-efficacy }\end{array}$ & $\begin{array}{c}\text { Modification in } \\
\text { received } \\
\text { interdependence due to } \\
\text { technological input }\end{array}$ & \\
\hline $\begin{array}{l}\text { Automating } \\
\text { technology }\end{array}$ & $\begin{array}{l}\text { Modification due to } \\
\text { total replacement of } \\
\text { humans }\end{array}$ & $\begin{array}{c}\text { Modification due to } \\
\text { either a sustained } \\
\text { attention or a passive } \\
\text { attitude }\end{array}$ & $\begin{array}{l}\text { Modification due to } \\
\text { fewer opportunities for } \\
\text { interactions }\end{array}$ & \\
\hline $\begin{array}{l}\text { Assisting } \\
\text { technology }\end{array}$ & Little influence & No proposition & No proposition & \\
\hline
\end{tabular}

Table 2.

Distribution of interviewees

\begin{tabular}{lcccc}
\hline Technology & Experts & Managers & Users & Total \\
\hline RPA & 1 & 1 & 2 & 4 \\
Drones & 2 & 0 & 3 & 5 \\
\hline
\end{tabular}




\begin{tabular}{ll}
\hline \multicolumn{1}{c}{ Assisting technologies } & \multicolumn{1}{c}{ Augmenting technologies } \\
\hline Drone & Structured artificial intelligence \\
Virtual reality & Big Data \\
Exoskeleton & Augmented reality \\
\hline \multicolumn{1}{c}{ Arresting technologies } & Automating technologies \\
\hline Blockchain & Unstructured artificial intelligence \\
Robotic Process Automation (RPA) & \\
3D printing & \\
\hline
\end{tabular}

Figure 1. Technological classification based on Murray's et al., (2020) $73 \times 38 \mathrm{~mm}(300 \times 300$ DPI $)$ 


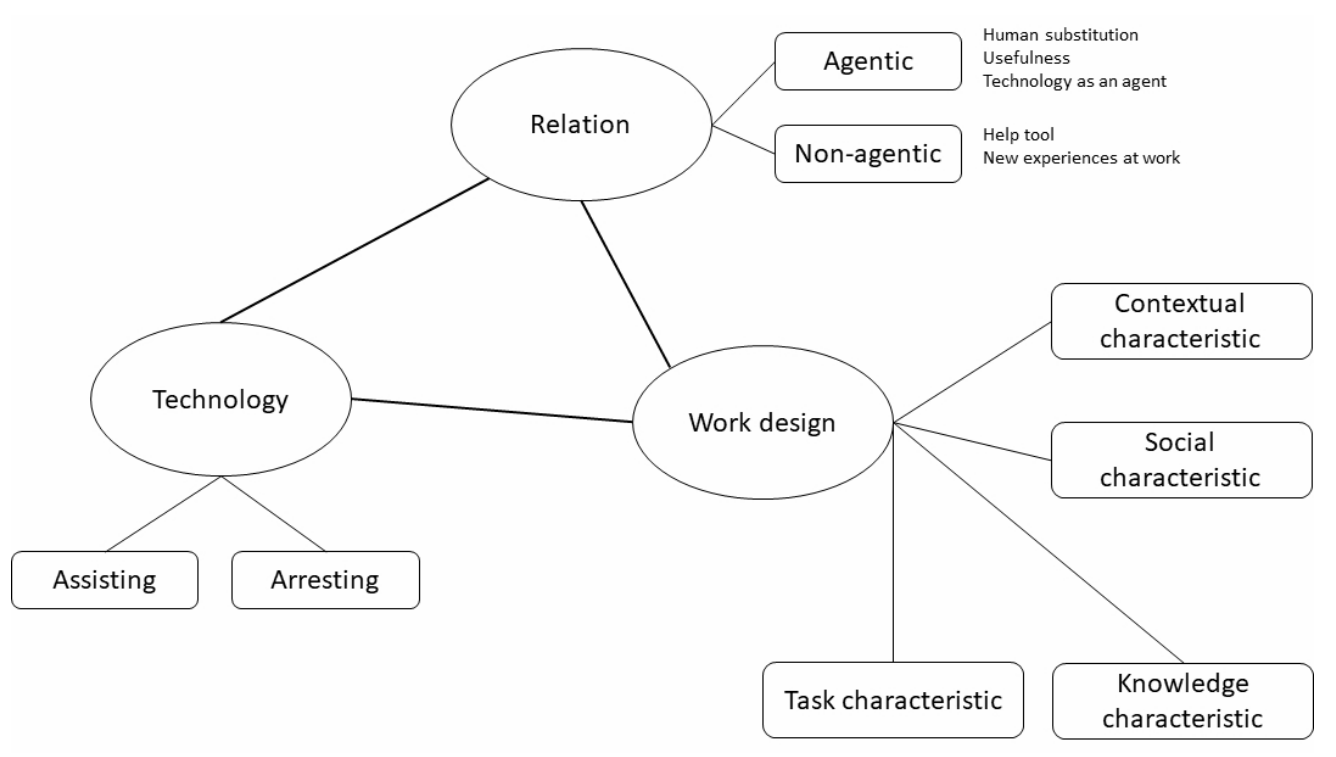

Figure 2. Thematic Map

$90 \times 51 \mathrm{~mm}(300 \times 300$ DPI $)$ 